\title{
Immune complex associated complications in the subacute phase of meningococcal disease: incidence and literature review
}

\author{
C A Goedvolk, I A von Rosenstiel, A P Bos
}

Arch Dis Child 2003;88:927-930

See end of article for authors' affiliations ......................

Correspondence to: Dr A P Bos, Emma Children's Hospital/AMC, PICU G8ZW, PO Box 22700, $1100 \mathrm{DE}$

Amsterdam, Netherlands; a.p.bos@amc.uva.nl

Accepted

3 January 2003

\begin{abstract}
Aim: To determine the incidence of immune complex associated complications (IAC) after severe meningococcal disease (SMD) in a group of Dutch children admitted to a paediatric intensive care unit (PICU).

Methods: Retrospective chart analysis and follow up of 130 survivors of SMD admitted to PICU. Signs of IAC, inflammatory parameters, and temperature profile were reviewed.

Results: Of 130 children with SMD, $20(15.3 \%)$ showed one or more of the three manifestations of IAC: 18 (13.8\%) developed arthritis (effusion, with or without erythema/arthralgia), 11 (8.4\%) vasculitis, and five (3.8\%) pleuritis. Eighteen of $20(90 \%)$ patients with IAC had a secondary rise in temperature; in patients with no IAC this was 48 of 110 (43.6\%). IAC was associated with leucocytosis in $82.3 \%$ versus $47.7 \%$ in patients without IAC, and with increased CRP in $86.6 \%$ versus $47.2 \%$ in patients without IAC. Leucocytes on admission were significantly lower in patients who would later develop IAC (mean 8.6 versus $13.8 \times 10^{\circ} / \mathrm{I}$ ).

Conclusion: IAC is a common complication of SMD, mainly occurring 4-10 days after systemic disease. IAC presents clinically as arthritis or vasculitis, mostly accompanied by secondary fever and raised inflammatory parameters.
\end{abstract}

M eningococcal disease is one of the most feared infections in children, due to its sometimes rapidly fatal course. Most of the research that has been done in recent years was directed at the pathophysiological mechanisms in the acute phase. Relatively little is known about complications occurring in the subacute phase (4-10 days after initial antibiotic treatment); the so-called type 3 immune complex hypersensitivity reactions, according to the classification of Gell and Coombs. Other examples of type 3 reactions include serum sickness and certain forms of glomerulonephritis. Antigen-antibody (immune) complexes cause an inflammatory reaction in tissue, leading to activation of complement and transmigration of polymorphonuclear leucocytes, resulting in tissue damage.

In meningococcal disease, type 3 reaction, further called immune complex associated complications (IAC), can present as arthritis, vasculitis, episcleritis, or pericarditis. ${ }^{1}$ Nephritis is a very rare presentation. It usually takes 4-10 days after the onset of disease for the first symptoms and signs of IAC to develop. Apart from the local manifestations, a recurrence of fever is often observed.

A search in PubMed using the keywords reactive arthritis, pericarditis, episcleritis, and pleuritis combined with meningococcal disease revealed only 16 case reports, ${ }^{2-17}$ and nine articles $^{118-25}$ containing larger patient groups on IAC since 1960. Since 1981 there have been no studies reported on large patient groups. Most of the articles were on adults with meningitis, who were not admitted to intensive care units. Only Schaad, ${ }^{23}$ Edwards and Baker, ${ }^{24}$ and Voss and colleagues ${ }^{25}$ studied children. In the past 20 years enormous progress has been made in the treatment of meningococcal disease in children, because of better paediatric intensive care unit (PICU) facilities. In our experience the incidence of reactive complications after meningococcal disease did not decline, thus the lack of recent publications is surprising.

The primary aim of this study was to determine the incidence of IAC after severe meningococcal disease in a group of Dutch children admitted to the PICU; the secondary aim was to determine whether there is any correlation between IAC and fever, leucocytosis, and other infection parameters. We compared our findings to the available literature.

\section{PATIENTS AND METHODS}

Between January 1993 and August 2000, 152 children were admitted to the PICU of the Emma children's hospital/AMC for treatment of severe meningococcal disease (SMD).

The records of all children still alive 24 hours after PICU admission were studied retrospectively for history of type 3 reactions. Furthermore, $\mathrm{C}$ reactive protein (CRP), erythrocyte sedimentation rate (ESR), maximal temperature, and white blood cell (WBC) count were noted for every day of hospital stay.

The diagnosis IAC was made clinically, according to the criteria summarised in table 1 . Other possible causes, such as secondary infection, were sufficiently excluded by additional investigations (synovial fluid aspiration, bone scan, $x$ ray examination).

A total of 133 children were still alive after 24 hours. Three patients were excluded because of lack of data, so the study group consisted of 130 children. All patients had the typical clinical presentation of meningococcal disease. In $70 \%$ of patients positive cultures were found. Most patients had systemic disease: sepsis with or without meningitis; some patients had only meningitis.

The following definitions were used:

- Fever: a temperature over $38.5^{\circ} \mathrm{C}$. For every 24 hours, maximal temperature was noted.

Abbreviations: CRP, $C$ reactive protein; $E S R$, erythrocyte sedimentation rate; IAC, immune complex associated complications; PICU, paediatric intensive care unit; $S M D$, severe meningococcal disease 
Table 1 Diagnostic criteria for IAC

\begin{tabular}{ll}
\hline Manifestation & Symptoms \\
\hline Arthritis & Arthralgia, joint swelling and redness, limitation of movement. Synovial fluid shows no bacteria, cultures remain negative. \\
Vasculitis & Pustular, bullous, nodular lesions or rash. \\
Pericarditis & Retrosternal pain, pericardial friction rub, ECG abnormalities, cardiac enlargement on $x$ ray/ultrasound. \\
Pleuritis & Pleural effusion on $x$ ray. Pain on inspiration. Impaired percussion. Pleural rub \\
\hline
\end{tabular}

Table 2 Patient characteristics of 130 evaluable patients

\begin{tabular}{ll}
\hline Patient & Characteristics \\
\hline Age & 3 y $8 \mathrm{mth}(2 \mathrm{mth}$ to 16 y $8 \mathrm{mth})$ \\
Males & $64(49.2 \%)$ \\
Deaths after 24 hours & $3(2.3 \%)$ \\
PRISM, mean (SD) & $13.41(10)$ \\
GMSPS, mean (SD) & $6.35(0.89)$ \\
Duration of PICU stay (days), median (range) & $4(1-46)$ \\
Duration of total stay (days), median (range) & $14.5(3-100)$ \\
\hline
\end{tabular}

- Leucocytosis: white blood cell count $\geqslant 20 \times 10^{9} / 1$.

- Increased ESR: $\geqslant 20 \mathrm{~mm}$ in the first hour.

- Increased CRP: $\geqslant 50 \mathrm{mg} / \mathrm{l}$.

- Secondary fever: a temperature over $38.5^{\circ} \mathrm{C}$ after at least one afebrile day, excluding day of admission.

The following demographic data were collected: age, sex, diagnosis, severity of disease score (PRISM, GMSPS), duration of PICU stay, duration of hospital stay, and serogroup typing.

Standard treatment for meningococcal disease in our PICU is ceftriaxone $100 \mathrm{mg} / \mathrm{kg}$ once daily as empiric antibiotic treatment; this is switched to penicillin $\mathrm{G}$ as soon as bacteriological confirmation and results of susceptibility testing of Neisseria meningitidis are available.

\section{Statistical analysis}

Statistical methods used were Fisher's exact test for nominal variables and $t$ test for comparing means of independent samples. Data were analysed with SPSS for Windows, release 8.0.0 (SPSS Inc., Chicago, IL). A p value $\leqslant 0.05$ was considered significant.

\section{RESULTS}

\section{Clinical parameters}

The study group consisted of 130 patients. Table 2 presents patient characteristics. One hundred patients had systemic disease (76.9\%; meningococcal sepsis with or without meningitis) and $30(23.1 \%)$ had only meningitis. All patients responded to antibiotic therapy and no patient had persisting infections.

In 52 patients $(40 \%)$, serogroup was recorded. In five patients serogroup typing revealed group C infection. No conclusions could be made about risk of IAC in different serogroup infections.

Twenty patients ( $15.3 \%$ ) developed IAC during the subacute phase. There was no correlation between the development of IAC and age, sex, PRISM, or GMSPS.

IAC presented as arthritis in $18(13.8 \%)$, vasculitis in 11 $(8.4 \%)$, and pleuritis in five $(3.8 \%)$. No clinical clues to indicate pericarditis or episcleritis were present in this study group. Twelve $(60 \%)$ patients had more then one manifestation of IAC. Pleuritis presented on median day 4 (range 3-15), arthritis on day 7 (2-15), and vasculitis on day 11 (3-16). Arthritis/arthralgia involved the knee joint in ten cases (55\%), the ankle in five cases $(27.7 \%)$, and the elbow in two $(11 \%)$. Six patients $(33 \%)$ had polyarthritis. Seventeen patients received some sort of additional treatment specifically aimed at IAC: ten were treated with additional antibiotics, four with NSAIDs, four received physiotherapy, and one patient was treated with steroids (dosage $\mathrm{l} \mathrm{mg} / \mathrm{kg} /$ day prednisone). Patients with IAC were admitted for longer than patients with an uncomplicated course, although not significantly so: 19.5 versus 13.8 days $(\mathrm{p}=0.08)$.

Sixty six of 130 patients $(50.8 \%)$ had secondary fever during the subacute phase of meningococcal disease. A secondary rise in temperature occurred in 18 of 20 patients in the IAC group $(90 \%)$ and in $48(43.6 \%)$ in the non-IAC group $(\mathrm{p}<0.001)$. The median duration of fever was 3 days (range $0-23)$.

Thirteen of 130 patients (10\%) developed an erythematous rash coinciding with the occurrence of secondary fever. These patients were not included in the IAC group as the clinical picture could not be differentiated from penicillin allergy.

\section{Laboratory parameters}

Fourteen of 17 (82.3\%) patients had leucocytosis at the time of IAC complaints in the subacute phase versus $43 / 90$ (47.7\%) patients without IAC at the same time $(p<0.05)$. Patients who later developed IAC had a significantly lower leucocyte count at admission: mean 8.6 (95\% CI 1.2 to 9.0) versus $13.8 \times 10^{9} / 1$.

CRP was measured in 15 patients with IAC at the time of complaints; 13 children had values $\geqslant 50 \mathrm{mg} / \mathrm{l}$. Of patients who did not develop IAC, $25 / 53(47.2 \%)$ had $C R P \geqslant 50 \mathrm{mg} / \mathrm{l}$ $(\mathrm{p}<0.01)$.

In all 13 patients with IAC where ESR was measured, it was increased beyond day 5. In patients without IAC, 46/56 $(82.1 \%)$ had increased ESR, which is not significantly different from patients with IAC.

\section{DISCUSSION}

Recently, little attention has been given to complications of meningococcal disease in the subacute phase. This so-called type 3 immune complex hypersensitivity reaction presents as arthritis, vasculitis, episcleritis, or pericarditis. In our study $15.3 \%$ of children admitted to PICU developed IAC; this is a high incidence compared to that reported in the literature (table 3). Risk factors for development of type 3 hypersensitivity reactions named in the literature are severe disease, ${ }^{124}$ serogroup C infections, ${ }^{26}$ and age (more IAC in adolescents or adults). ${ }^{123}{ }^{24}$ The fact that our patients were more severely ill compared to previous study groups may explain the higher 
Table 3 Incidence of IAC reported in the literature compared to this study

\begin{tabular}{lll}
\hline Manifestation & Literature (\%) & This study (\%) \\
\hline Total & $6-11^{1182324}$ & 15.3 \\
Arthritis & $1.6-16.6^{112} 18202123-25$ & 13.8 \\
Vasculitis & $1.7-4.7^{124}$ & 8.4 \\
Pericarditis & $0.9-19^{1920}$ & - \\
Pleuritis & - & 3.8 \\
\hline
\end{tabular}

incidence of IAC. Most of our patients (76.9\%) suffered from systemic disease (sepsis), in contrast to previous studies where most patients only had meningitis. This may be another explanation for the higher incidence of IAC, since these patients have a higher antigen load in the acute phase of the disease with a higher risk of developing IAC. No investigations into immune complexes and complement in blood and synovial fluid have been done in our study.

Arthritis was the most common presentation of IAC (13.8\%), similar to that reported in the literature (table 3 ). As in the literature, the most involved joint in our patients with reactive arthritis was the knee, followed by the elbow and ankle. The time at which symptoms occured in our study group, though slightly later, is comparable to that reported in the literature.

Pleuritis or pleural effusion is described in four case reports. ${ }^{6}{ }^{16} 1819$ No other references could be found, but it seems likely that the same mechanism that causes pericarditis can also cause pleuritis.

Edwards and Baker ${ }^{24}$ found shock, purpura, leucocytosis $\geqslant 20 \times 10^{9} / 1$, or leucopenia $\leqslant 5 \times 10^{9} / 1$ on admission and persisting fever to be prognostic for IAC. In our group no correlation was found between leucocytosis on admission and IAC, but we did find a correlation with leucopenia on admission. We also found an association between IAC and leucocytosis in the subacute phase.

We found the occurrence of IAC is associated with secondary fever and secondary increased ESR and CRP, so persisting fever as mentioned by Edwards and Baker ${ }^{24}$ is probably not a prognostic sign for development of IAC but a symptom of IAC per se, sometimes occurring before symptoms of arthritis/ vasculitis.

In our group a high frequency of erythematous, nonpurpuric rash was found $(10 \%)$. Sometimes this was diagnosed as suspected penicillin allergy, but often diagnosis was unclear and symptoms subsided spontaneously. The frequency is too high to attribute all these cases to penicillin allergy (incidence normally $1 \%$ ). We would like to argue that this may well be a manifestation of IAC, as van Deuren and colleagues $^{27}$ suggested, yielding an even higher incidence of $25 \%$ of IAC in our cohort.

\section{Implications for clinical practice}

This study shows that the incidence of secondary fever and raised inflammatory parameters in IAC is high; unfortunately this fact can not be used as a diagnostic tool since other complications like secondary infection and tissue necrosis are also accompanied by raised inflammatory parameters.

In patients with secondary fever or raised inflammatory parameters after SMD, IAC should always be considered in differential diagnosis. Symptoms and signs of IAC should be looked for on physical examination, since they can easily be missed, especially in severely ill patients. Fever can also be a sign of other complications such as secondary infection, subdural effusion, and persisting infection. In these cases extensive physical examination will reveal clues for the diagnosis, without the necessity of extensive additional investigations.
Primary meningococcal arthritis and allergic reaction to medication have to be excluded, preferably with as little additional and invasive diagnostics as possible. Primary meningococcal arthritis is a purulent arthritis with positive cultures that may occur without meningococcaemia.

Patients with IAC were admitted for a longer period then patients with an uncomplicated course. The difference is not significant $(\mathrm{p}=0.08)$, but studying the charts of patients with IAC, it was often noted that children were hospitalised longer for antibiotic treatment or physiotherapy and additional investigations (bone scans). These measures are ineffective or unnecessary in IAC.

According to the literature, specific treatment for arthritis is not indicated, except for pain relief. The prognosis is excellent. $^{15} 2324$

Contrary to other immune complex associated complications, pericarditis has a substantial mortality related to cardiac tamponade. Occasionally the effusion clears spontaneously, but usually treatment is required. Therapy consists of salicylates, steroids, pericardiocentesis, or a combination of these. $^{6}$

\section{Conclusions}

IAC is a common complication of meningococcal infections in children. Arthritis is the most common manifestation of IAC. Apart from clinical symptoms, IAC presents with fever, leucocytosis, and increased CRP. IAC often leads to prolonged hospital stay and unnecessary diagnostic procedures and treatment.

In comparison to reports in the literature, this study shows that the incidence of reactive complications after meningococcal disease has not declined despite better treatment modalities, and that it also occurs in patients with sepsis.

Because of the limitations of a retrospective study, we recommend that a prospective study is done, in order to investigate risk factors, serogroup typing, and complement profile in IAC.

\section{Authors' affiliations}

C A Goedvolk, I A von Rosenstiel, A P Bos, Department of Pediatric Intensive Care, Academic Medical Center, University of Amsterdam, Emma Children's Hospital/AMC, Amsterdam, Netherlands

\section{REFERENCES}

1 Whittle HC, Abdullahi MT, Fakunle FA, et al. Allergic complications of meningococcal disease I-clinical aspects. BM 1973;2:733-7.

2 Saslaw S, Diserens RV. Purulent pericardial effusion complicating meningococcal meningitis. N Engl J Med 1960;24:1074-5

3 Penny JL, Grace WJ, Kennedy RJ. Meningococcic pericarditis. A case report and review of the literature. Am J Cardiol 1966;18:281-5. 4 Williams DN, Geddes AM. Meningococcal meningitis complicated by pericarditis, panophthalmitis and arthritis. BM 1970;2:93.

5 O'Connell B. Pericarditis following meningococcic meningitis. Am J Dis Child 1973;126:265-7.

6 Miller GC, Calhoun Witham A. Delayed febrile pleuropericarditis after sepsis. A possible analogue of the postcardiotomy syndrome. Ann Intern Med 1973:79:194-7.

7 Keeling PWN, Aston NO, Croft DN. Steroid-responsive vasculitis and arthritis in meningococcal septicaemia. Postgrad Med J 1980;56:800-1.

8 Jarret MP, Moses S, Barland P, et al. Articular complications of meningococcal meningitis. An immune complex disorder. Arch Intern Med 1980;140:1665-6.

9 Stephani U, Bleckmann H. Rare complications in a case of generalized meningococcal disease: immunologic reaction versus bacterial metastasis. Infection 1982;10:23-7.

10 Vega LA. Acute meningococcemia with DIC, septic shock complicated by hypersensitivity monoarthritis (case report in a 20-month old patient with complete clinical recovery). W Virg Med J 1984;80:6-9.

11 Rosen MS, Meyers AR, Dickey B. Meningococcemia presenting as septic arthritis, pericarditis, and tenosynovitis. Arthritis Rheum 1985;28:576-8.

12 Greenwood BM, Mohammed I, Whittle HC. Immune complexes and the pathogenesis of meningococcal arthritis. Clin Exp Immunol 1985;59:513-19.

13 Olivieri I, Pifferi $M$, Ceccarelli $M$, et al. Erosive immune complex-mediated arthritis associated with meningococcal meningitis. Clin Rheumatol 1986:5:531-4.

14 Elechi CA. A case of meningococcal meningitis with unusual complications. Trop Geogr Med 1988;40:353-5. 
15 Postema RR, Brinkman JG, Neyens HJ. Arthritis als complicatie van acute meningokokkeninfectie. Ned Tijdschr Geneeskd 1993;137: 1152-4

16 Moss W, Prince A. Pericarditis complicating meningococcal meningitis in a 7-month-old boy. Clin Pediatr 1994;33:169-71.

17 Beggs S, Marks M. Meningococcal pericarditis in a 2-year-old child: reactive or infectious? J Paediatr Child Health 2000;36:606-8.

18 Wolf RE, Birbara CA. Meningococcal infections at an army training center. Am J Med 1968:44:243-5.

19 Morse JR, Oretsky MI, Hudson JA. Pericarditis as a complication of meningococcal meningitis. Ann Intern Med 1971;74:212-17.

20 Dixon LM, Hess RJ, Smith AE. Meningococcal arthritis. Military Medicine 1972;12:425-7.

21 Whittle HC, Greenwood BM, Davidson N, et al. Meningococcal antigen in diagnosis and treatment of group A meningococcal infections. Am Med 1975:58:823-8
22 Greenwood BM, Onyewotu II, Whittle HC. Complement and meningococcal infection. BM 1976;1:797-9.

23 Schaad UB. Arthritis in disease due to Neisseria meningitidis. Rev Infect Dis 1980;2:880-7.

24 Edwards MS, Baker CJ. Complications and sequelae of meningococcal infections in children.J Pediatr 1981;99:540-5.

25 Voss L, Lennon D, Sinclair J. The clinical features of paediatric meningococcal disease Auckland, 1985-87. N Z Med J 1989;102:243-5

26 Finkelstein $Y$, Adler $Y$, Nussinovitch $M$, et al. A new classification for pericarditis associated with meningococcal infection. Eur J Pediatr 1997; 156:585-8.

27 Van Deuren M, Brandtzaeg P, van der Meer JWM. Update on meningococcal disease with emphasis on pathogenesis and clinical management. Clin Microbiol Rev 2000;13:144-6.

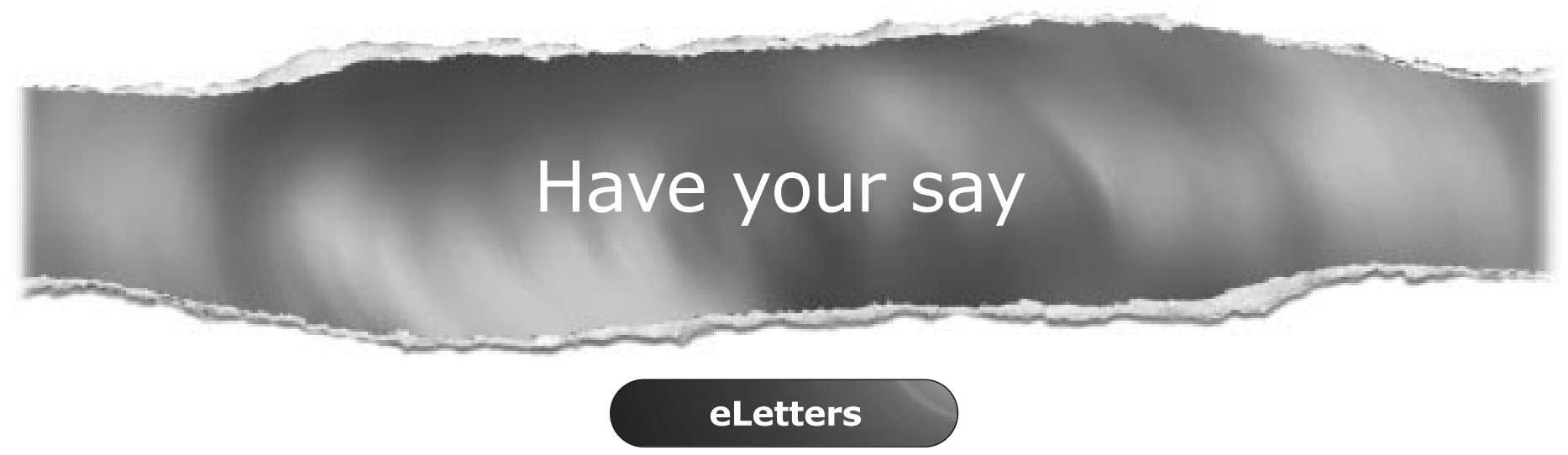

If you wish to comment on any article published in Archives of Disease in Childhood you can send an eLetter using the eLetters link at the beginning of each article. Your response will be posted on Archives of Disease in Childhood online within a few days of receipt (subject to editorial screening).

www.archdischild.com 